Supporting Information for

\title{
Nitric oxide-generating anti-platelet polyurethane surfaces with multiple additional biofunctions via cyclodextrin-based host-guest interactions
}

Sheng Jin, Jialei Huang, Xianshuang Chen, Hao Gu, Dan Li,* Aiyang Zhang, Xiaoli Liu, * and Hong Chen

State and Local Joint Engineering Laboratory for Novel Functional Polymeric Materials, College of Chemistry, Chemical Engineering and Materials Science, Soochow University, 199 Ren-Ai Road, Suzhou 215123, P. R. China.

\section{Corresponding Author}

*Tel: +86-512-65880827; Fax: +86-512-65880583;

E-mail: lidan@suda.edu.cn (D. Li); liuxiaoli@suda.edu.cn (X. Liu). 


\section{Materials}

Tecothane polyurethane (TT-1095A) was from Thermedics (Wilmington, MA, USA). 2,2'-Azoisobutyronitrile (AIBN) was from Tokyo Chemical Industry Company (Shanghai, China) and recrystallized prior to use. 2-hydroxyethyl methacrylate (HEMA, 97\%), from Acros Organics, was purified using an inhibitor removal column. 6-(4-sulfonylmethyl-1 $H$-[1,2,3]triazol-1-yl)-6-deoxy- $\beta$-cyclodextrin $\quad(\mathrm{CD}-\mathrm{S}),{ }^{1} \quad$ a $\beta$-cyclodextrin derivative bearing seven lysine ligands $(\mathrm{CD}-\mathrm{L})^{2}$ and 1-adamantan-1-ylmethyl methacrylate (AdaMA) ${ }^{3}$ were synthesized as described in previous reports. Copper (I) bromide (CuBr, 98\%), $N, N, N$, $N$,', $N$ ''-pentamethyldiethylenetriamine (PMDETA, 99\%), $\beta$-cyclodextrin, methacryloyl chloride (stabilized with hydroquinone monomethyl ether), S-nitrosoglutathione (GSNO), L-glutathione (GSH), N,N'-carbonyldiimidazole (CDI), Actin-Tracker Green (Phalloidin-FITC) and 4',6-diamidino-2-phenylindole (DAPI) were from Sigma-Aldrich Chemical Company (USA). Fibrinogen was from Calbiochem (La Jolla, CA). Plasminogen was from Enzyme Research Laboratories (South Bend, IN, USA). Tissue plasminogen activator (t-PA) was from Genentech (San Francisco, CA). $\mathrm{Na}^{125}$ I was from Chengdu Gaotong Isotope Co., Ltd (Chengdu, China). Human umbilical vein endothelial cells (HUVECs), human umbilical vein smooth muscle cells (HUVSMCs), endothelial cell medium (ECM) and smooth muscle cell medium (SMCM) 
were from ScienCell (USA). Ethylenediaminetetraacetic acid (EDTA) and all organic solvents were from Sinopharm Chemical Reagent Co. (Shanghai, China). The organic solvents were purified according to standard methods. Human plasma and platelet rich human plasma (PRP) were from the Suzhou Blood Center (Suzhou, China). Griess reagent nitric oxide assay kit was from Beyotime Biotech. (Shanghai, China).

\section{Characterization}

All prepared compounds were analyzed by ${ }^{1} \mathrm{H}$ nuclear magnetic resonance spectroscopy ( ${ }^{1} \mathrm{H}$ NMR, Varian Inova $400 \mathrm{MHz}$ instrument, USA) with $\mathrm{D}_{2} \mathrm{O}, \mathrm{CD}_{3} \mathrm{OD}$ or DMSO- $\mathrm{d}_{6}$ as solvent. Static water contact angles were measured using an SL200C optical contact angle meter (Solon Information Technology Co., Ltd., China) at $25{ }^{\circ} \mathrm{C}$. The surface chemical compositions were determined by X-ray photoelectron spectroscopy (XPS) using an ESCALAB 250 XI X-ray photoelectron spectrometer (Thermo Scientific, USA). After dehydration, the platelets on surfaces were examined using field-emission scanning electron microscopy (FESEM, S4700, Hitachi, Japan). The cells on the surfaces were examined using a fluorescence microscope (Olympus IX71 Carl Zeiss, Germany). The fluorescence images were analyzed with Image-Pro software.

\section{References}

(1) Lyu, Z.; Shi, X.; Lei, J.; Yuan, Y.; Yuan, L.; Yu, Q.; Chen, H. Promoting Neural 
Differentiation of Embryonic Stem Cells Using $\beta$-Cyclodextrin Sulfonate. J. Mater. Chem. B 2017, 5, 1896-1900, DOI: 10.1039/C6TB02572B.

(2) Gu, H.; Chen, X.; Yu, Q.; Liu, X.; Zhan, W.; Chen, H.; Brash, J.L. A Multifunctional Surface for Blood Contact with Fibrinolytic Activity, Ability to Promote Endothelial Cell Adhesion and Inhibit Smooth Muscle Cell Adhesion. J. Mater. Chem. B 2017, 5, 604-611, DOI: $10.1039 / \mathrm{C} 6 \mathrm{~TB} 02808 \mathrm{~J}$.

(3) Zhan, W.; Shi, X.; Yu, Q.; Lyu, Z.; Cao, L.; Du, H.; Liu, Q.; Wang, X.; Chen, G.; Li, D.; Brash, J.L.; Chen, H. Bioinspired Blood Compatible Surface Having Combined Fibrinolytic and Vascular Endothelium-Like Properties via a Sequential Coimmobilization Strategy. Adv. Funct. Mater. 2015, 25, 5206-5213, DOI: 10.1002/adfm.201501642. 